\title{
Visual search for apparent-length targets is modulated by the Müller-Lyer illusion
}

\author{
ASTRID BUSCH* and HERMANN J. MÜLLER \\ Department of Psychology, Ludwig-Maximilians-University, Munich, Germany
}

Received 28 May 2003; revised 30 October 2003; accepted 19 Dcember 2003

\begin{abstract}
Is apparent object size represented in pre-attentive vision and can it influence visual search for size-defined targets in a spatially parallel manner? This question was investigated, using the Müller-Lyer illusion. Observers searched for a target line that was longer than the distractor lines. Test lines could be presented without context arrows (control); be adjoined by obtuse-angle context arrows (arrow heads pointing inward), making the lines appear longer; or by acute-angle arrows (heads pointing outward), making the lines appear shorter. These apparent-length modulations were larger for the target than for the distractor lines, thereby increasing and, respectively, decreasing the targetdistractor length contrast. In line with these changes in contrast, target detection was found to be expedited by obtuse-angle arrows and impeded by acute-angle arrows, independently of the number of elements in the display. This finding provides further evidence for the pre-attentive processing of apparent object size.
\end{abstract}

Keywords: Pre-attentive processing; apparent length; Müller-Lyer illusion.

\section{VISUAL SEARCH FOR FEATURE TARGETS}

Visual information processing has been proposed to involve two stages: an early pre-attentive stage followed by a later attention-dependent stage (e.g. Neisser, 1967; Treisman, 1988; Treisman and Gelade, 1980). One experimental paradigm to study early visual processing is visual search. In the typical visual search task, observers are presented with displays consisting of varying numbers of objects (number $=$ 'display size'), and their task is to decide whether or not a display contains a prespecified target object. A typical finding is that detection reaction times (RTs) do not increase with increasing display size when the target differs from the non-targets ('distractors') in a distinctive visual attribute, or 'basic feature'. Phenomenally,

\footnotetext{
*To whom correspondence should be addressed at Department Psychologie, Ludwig-MaximiliansUniversität München, Leopoldstrasse 13, D-80802 München/Munich, Germany. E-mail: abusch@psy.uni-muenchen.de
} 
such feature targets appear to 'pop out' of the display. Such basic-feature searches are interpreted as reflecting a spatially 'parallel' (Treisman and Gelade, 1980) or 'efficient' (Wolfe, 1998) search process, with target detection mediated by unique activation in a map of detectors tuned to the particular feature that characterizes the target within its defining (feature) dimension.

It is well established that visual search for a variety of feature targets can operate efficiently, for example, search for orientation-, color-, motion-defined targets (see Wolfe, 1998, for a review). Several studies have also demonstrated efficient search for size-defined targets (e.g. Bilsky and Wolfe, 1995; Busch and Müller, 2004; Duncan and Humphreys, 1992; Müller et al., 1995; Quinlan and Humphreys, 1987; Stuart et al., 1993; Treisman and Gelade, 1980; Treisman and Gormican, 1988), providing evidence for the idea that object size is computed and represented preattentively.

From a number of studies, it is known that the features supporting efficient search can be more complex than primitive (simple) visual attributes. In particular, there is evidence that secondary-depth information, that is, cues to the three-dimensional shape (e.g. y-junction information) and spatial layout of objects (e.g. occlusion relations) may be derived pre-attentively (e.g. Aks and Enns, 1992; Enns, 1992; Enns and Rensink, 1990a, 1990b, 1991; Epstein and Babler, 1990; Epstein and Broota, 1986; Epstein et al., 1992; Rensink and Enns, 1995). These findings provide evidence that, in early visual processing, not only image-based (two-dimensional), but also scene-based (three-dimensional) features are computed and represented pre-attentively (see also Kleffner and Ramachandran, 1992; Ramachandran, 1988).

\section{VISUAL SEARCH FOR APPARENT-SIZE TARGETS}

With regard to object size, a number of studies have suggested that not only the image-based ('retinal') size, but also the perceived ('apparent') size of objects is computed pre-attentively. The distinction between retinal and apparent size refers to the phenomenon that an object of a given size that is perceived to be 'far' from the observer appears larger than an object of identical size that is perceived to be 'close' to the observer. Thus, apparent object size takes into account the (perceived) distance between the observer and the object. Most studies of the size representation of objects have varied only their retinal size. However, in several recent studies (e.g. Aks and Enns, 1996; Busch and Müller, 2004; Found and Müller, 2001; Humphreys et al., 1994), the apparent object size was manipulated, too. These studies have provided some evidence that apparent object size is derived and represented preattentively. For instance, Busch and Müller (2004) modulated the apparent size of target and distractor objects by using the geometric-optical Ebbinghaus illusion. In the original version of this illusion (Ebbinghaus, 1913), an inner 'test' circle is surrounded by outer 'context' circles. Smaller context circles make the test circle appear larger, and larger context circles make it appear smaller, relative to a test circle presented on its own. Busch and Müller found that, when the target's apparent 
size was enhanced by operation of the Ebbinghaus illusion relative to the size of the distractors, detection was expedited in comparison with control conditions in which efficient search was based solely on comparisons of the target and distractors' retinal sizes. This finding, together with those cited above, provides some evidence of preattentive processing of apparent-size information.

One problem that besets the study of Busch and Müller lies in the origin of the Ebbinghaus illusion, coupled with the demands of a visual-search task. To produce an apparent-size manipulation of the inner 'test' circle (i.e. a perceptible 'distortion' of its absolute size), it is necessary to 'integrate', in some sense, the inner test circle with the surrounding context circles into a coherent configuration. Yet, at the same time, to perform the visual search task, it is necessary to focus processing on one of the configuration parts, the inner circle, as the task-relevant (test) object. Müller and Busch (2003) provided evidence that the latter involves active suppression of the context circles, which, besides modulating the test circles' apparent size, are (featurally) similar to the test objects and so interfere with search performance (Duncan and Humphreys, 1989). As a result, effects of the Ebbinghaus apparentsize modulation in visual search are partially masked by suppression of the context circles.

Therefore, in the present study, a series of experiments was conducted in which display objects' apparent size was modulated by the Müller-Lyer illusion (MüllerLyer, 1889). This illusion arises if the lengths of test lines that are adjoined by context arrows of different angles have to be judged. A test line adjoined by arrows of acute angle (arrow head pointing outward from the test line) appears smaller than a comparison line of identical length, whereas a test line adjoined by arrows of obtuse angle (arrow head pointing inward to the test line) appears longer (see Fig. 1). This geometric-optical illusion was used to support and generalize the finding of Busch and Müller (2004) that apparent object size can modulate the efficiency of visual search for a size-defined target. Because the context arrows in the MüllerLyer illusion are featurally (i.e. in terms of component line orientations) dissimilar to the test lines, they were expected not to interfere with search performance (Duncan and Humphreys, 1989); that is, they would not need to be suppressed, in contrast to the context circles in Ebbinghaus configurations. Analogously to the experiments of Busch and Müller (2004), observers were presented with search displays that consisted of varying numbers of Müller-Lyer configurations. Observers had to search for a longer target line amongst smaller distractor lines. The test lines' apparent size was modulated by context arrows of either obtuse or acute angle.

Pertinent to the present experiments, Rensink and Enns (1995) investigated whether Müller-Lyer stimuli are processed as single line segments or as complete configurations. Their observers had to search for a target configuration with one

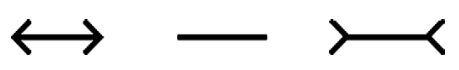

Figure 1. Illustration of the Müller-Lyer illusion. 
type of arrows (e.g. obtuse angles) amongst a number of distractor configurations with the other type of arrows (e.g. acute angles), with target and distractor configurations having (1) either different overall length and same test line length (condition 'different-overall'), or (2) same overall length and different test line length (condition 'different-segment'), or (3) different overall length and different test line length (condition 'different-combined') (e.g. Experiment 1). Observers could search for the target in three different ways: search for a different overall assembly, search for a different test segment, or search for a different junction between test line and context arrows. The results revealed search to be efficient in the 'different-overall' condition (target-present search rates about $5 \mathrm{~ms} /$ item); relatively efficient in 'different-combined' condition (search rates smaller than $10 \mathrm{~ms} /$ item); and less efficient in the 'different-segment' condition (search rates about $15 \mathrm{~ms} / \mathrm{item}$ ). Rensink and Enns took this pattern of results to indicate that 'rapid' visual search is based not on 'contour fragments', but rather on 'complete configurations' produced by low-level grouping processes: "Visual search cannot access the primitive [object] elements formed at the earliest stages of visual processing; rather, it can access only higher level, more ecologically relevant structures" (p. 101).

Although using similar (Müller-Lyer) stimuli, the present experiments were designed to examine a question different from that addressed by Rensink and Enns (1995). Whereas they investigated whether test lines and context arrows of MüllerLyer configurations are processed as grouped objects, the present experiments examined whether context arrows influence the test lines' perceived lengths at an early, pre-attentive, rather than a later, attentional, stage of processing (that is, how apparent length is computed in relation to retinal length). Thus, in contrast to Rensink and Enns' task, the presence of a target 'configuration' in the display could not be detected on the basis of a unique type of context arrow; and observers were forced to direct attention towards the task-relevant test lines to discern whether a longer (i.e. target) line was present in the display (whereas the context arrows could be ignored as task-irrelevant). One conceivable strategy for observers to solve this task would be to 'break down' the Müller-Lyer configurations and derive measures of the test lines' retinal lengths. Alternatively, extrapolating from Rensink and Enns' findings, observers might have to take the context arrows into account to compute the test lines' apparent lengths as the basis of the required decision. If the latter alternative could be shown to be true, the present experiments would provide an important extension to the findings of Rensink and Enns.

Although not of prime concern for the present study, it is interesting to briefly consider theoretical accounts of the Müller-Lyer illusion. Gregory (1966) attributed the illusion to a mis-application of size constancy scaling: with lines adjoined by obtuse-angle arrows being perceived as further away and, therefore, longer than lines adjoined by acute-angle arrows. However, DeLucia and Hochberg (1985, 1986, 1991) demonstrated that the illusion also occurs with 'three-dimensional' displays in which the to-be-judged spaces (lengths) between the sets of obtuse and 
acute arrow fins are not perceived to be at the different depths. This argues against a depth-based interpretation being crucial for the illusion. Subsequently, Day (1989, 1990) proposed that it is not a misinterpretation of depth information that gives rise to the illusion, but rather conflicting length cues (actual length of to-be-judged line and overall length of configuration) that are integrated to form a compromise perception of length (see also Rensink and Enns, 1995). On this account, the illusion exploited in the present study is set apart from the secondary-depth information that has, hitherto, been manipulated to examine the influence of apparent-size coding on visual-search performance (e.g. Aks and Enns, 1996; Found and Müller, 2001; Humphreys et al., 1994).

\section{RATIONALE AND OVERVIEW OF EXPERIMENTS}

The present experiments examined whether the Busch and Müller's (2004) findings with the Ebbinghaus illusion extend to the Müller-Lyer illusion. Observers were presented with displays that consisted of varying numbers of horizontally oriented Müller-Lyer configurations (test lines plus context arrows) and/or, respectively, simple test lines (without context arrows). Observers' task was to discern the presence of a longer target test line $(26 \mathrm{~mm})$ amongst shorter distractor test lines $(13 \mathrm{~mm})$ and make an appropriate target-present/absent decision.

Based on a preliminary, psychophysical experiment (Experiment 1), the parameters of the Müller-Lyer configurations presented in the visual-search experiments (Experiments 2 and 3) were chosen so as to result in differential mis-judgments of target and distractor configurations with equal-size and equal-angled context arrows. In particular, relative to test stimuli without context arrows (length difference of $26-13=13 \mathrm{~mm}$; subjective difference of $12.94 \mathrm{~mm}$ ), context arrows of obtuse angle increased the apparent length of a target line more than that of distractor lines (29.54 [26.00] vs. 15.74 [13.00] mm; subjective difference of $13.80 \mathrm{~mm})$. In contrast, context arrows of acute angle decreased the apparent length of a target line more than that of distractor lines $(23.75$ [26.00] vs. 11.23 [13.00] mm; subjective difference of $12.52 \mathrm{~mm}$ ).

Thus, importantly, the apparent-length manipulation - magnification by obtuseangle arrows and, respectively, de-magnification by acute-angle arrows - was absolutely larger for the (longer) target than for the (shorter) distractor lines, increasing and, respectively, decreasing the apparent-length difference between the target and distractors. Consequently, if observers, in the search task, use the apparent lengths of the test lines, more precisely, the apparent-length difference between the target and distractor lines (rather than their retinal lengths), to discern the presence of a target, visual search performance should be differentially influenced by the apparent-length manipulations. For conditions in which the apparent-length difference between target and distractor configurations is increased (i.e. with obtuseangle context arrows), target detection should be expedited (overall faster search RTs, shorter base RTs, and/or faster search rates). In contrast, for conditions in 
which the apparent-size difference is decreased (i.e. with acute-angle context arrows), target detection should be impeded (overall slower search RTs, longer base $\mathrm{RTs}$, and/or slower search rates).

With overall 'efficient' search performance (i.e. search RTs independent of the number of test lines in the display), the beneficial and, respectively, harmful effects of the apparent-length manipulation were expected to become manifest mainly in terms of overall RT and base RT effects. Such a pattern of results would support the hypothesis that apparent-length information is processed pre-attentively, spatially 'in parallel' across the display.

These predictions, which mirror the results of Busch and Müller's (2004) study with the Ebbinghaus illusion, were confirmed in Experiment 2 (homogeneous displays consisting of Müller-Lyer configurations of one context arrow type only) and Experiment 3 (heterogeneous displays) (see Note 1).

\section{EXPERIMENTS 1 AND 2: RATIONALE}

The rationale of this set of experiments was as follows. First, a psychophysical experiment (Experiment 1) was conducted to determine the size of the line length mis-judgments (i.e. the apparent length modulation by the Müller-Lyer illusion) for target and distractor configurations under acute- and obtuse-angle context arrow conditions. To estimate the apparent line lengths, observers were presented with displays containing one Müller-Lyer configuration and one variable-length comparison line without context arrows and asked to indicate which line appeared longer. This method permitted interpolation of the (retinal) length of the comparison line (without context arrows) that corresponded to the apparent length of the test line (with context arrows).

The lengths thus determined were then introduced as 'adapted' control conditions (without context arrows) in a visual search experiment (Experiment 2), to directly compare search performance under test conditions of purely retinal and (retinal plus) apparent lengths (adapted control vs. context arrow conditions). If the detection RTs were 'equivalent' under the two test conditions (e.g. equivalent target detection RTs under the obtuse-angle context arrow and the corresponding adapted control condition), this would support the case that the Müller-Lyer illusion is computed spatially in parallel for all display configurations, modulating target saliency (i.e. the length contrast between target and distractors) and, consequently, detection RTs.

\section{EXPERIMENT 1}

Experiment 1 was designed to determine the size of the line length mis-judgments, for target and distractor lines, under acute- and obtuse-angle context arrow conditions. 


\section{Method}

Participants. Eighteen observers (17 female; ages ranging from 19 to 35 years) participated in two experimental sessions. All reported normal or corrected-tonormal vision. Observer were paid at a rate of 8 Euro per hour for their participation.

Apparatus. Displays were presented on a $17^{\prime \prime}$ monitor driven by a personal computer. The laboratory was dimly illuminated. Responses were recorded from an ERTS keyboard. Observers viewed the display from a distance of $60 \mathrm{~cm}$.

Stimuli and task. Each stimulus display consisted of one Müller-Lyer configuration and one comparison line, positioned $4.50 \mathrm{~cm}$ to the left and the right of the screen center, with lateral position of the test configuration and comparison line varying randomly across trials (stimuli black, background white). The Müller-Lyer configuration was a test line either 13 or $26 \mathrm{~mm}$ in length, adjoined by context arrows of acute $\left(45^{\circ}\right)$ or obtuse $\left(135^{\circ}\right)$ angle or without context arrows (control conditions in the following experiments). The length of the comparison line was varied in $0.5-\mathrm{mm}$ steps within the ranges $5.5-20.5 \mathrm{~mm}$ and, respectively, $18.5-33.5 \mathrm{~mm}$, depending on the length of the test line (13 or $26 \mathrm{~mm}$ ). Observers were instructed to indicate which of the two lines - the test line in the Müller-Lyer configuration or the comparison line - appeared longer, by pressing a corresponding, left or right, response button (non-speeded response).

Design and procedure. The independent variables in Experiment 1 were: length of test line $(13,26 \mathrm{~mm})$, context arrows (zero, acute, or obtuse), and length of comparison line (varying in 31 steps of $0.5 \mathrm{~mm}$ ). Observers performed the required judgments with displays of all 186 factorial combinations of these variables, presented in random order across an experimental session (10 trials per combination per session, such that, overall, there were 20 repetitions of each of the experimental conditions across both sessions). Each session consisted of 30 blocks of 62 experimental trials and took approximately 60 minutes to complete.

\section{Results}

Figure 2 presents the group mean frequency of 'test-line-longer-than-comparisonline' responses as a function of context arrows, separately for both test line length conditions. That length of comparison line at which the frequency of 'test-linelonger-than-comparison-line' responses was $50 \%$ was taken as representing the apparent length of the test line in each experimental condition by linear interpolation of the exact value (point of subjective equality, PSE). [Given the near-linear decrease of the response frequency/comparison line length functions around the $50 \%$ point (see Fig. 2), simple linear interpolation seemed sufficiently accurate to estimate the PSE.] 


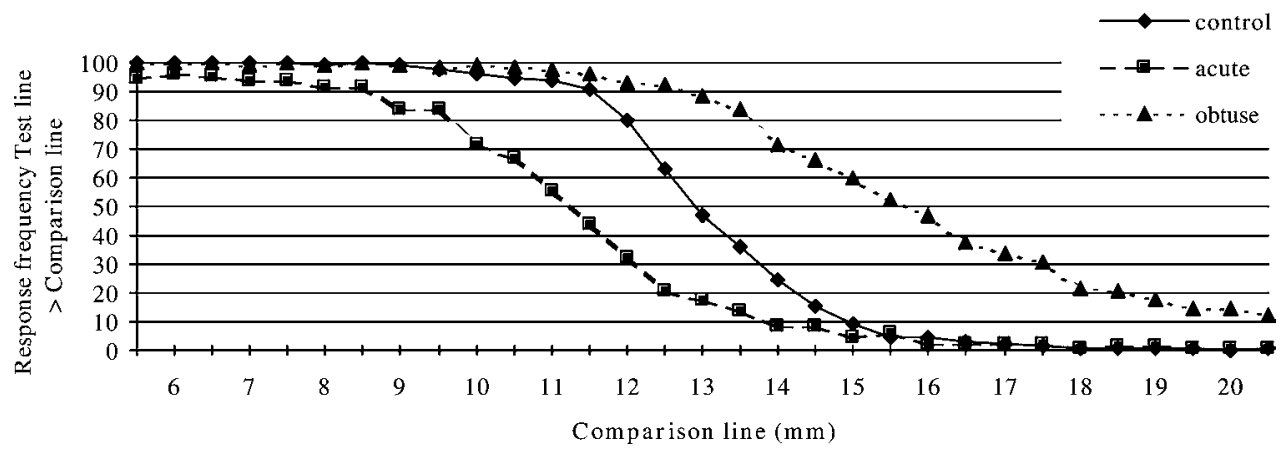

(A)

Test line $26 \mathrm{~mm}$

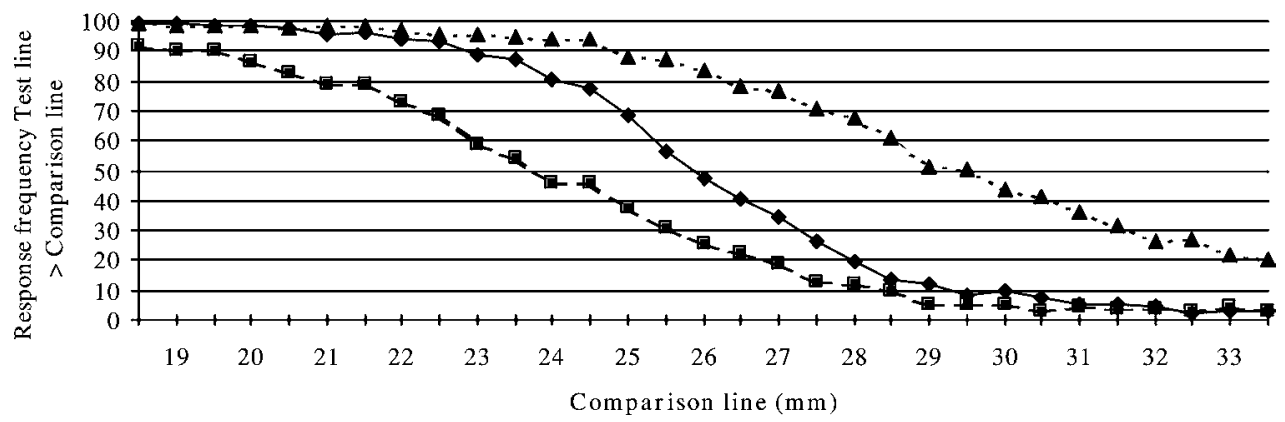

(B)

Figure 2. Mean frequency of 'test-line-appears-longer-than-comparison-line' responses (in percent) in Experiment 1, for $13 \mathrm{~mm}$ (distractor; Fig. 2A) and $26 \mathrm{~mm}$ (target; Fig. 2B) test lines, respectively. Control, acute-angle, and obtuse-angle arrow context conditions are represented by solid, dashed, and dotted lines, respectively.

As can be seen, a Müller-Lyer configuration with acute-angle context arrows appeared shorter than a configuration with obtuse-angle context arrows. An analysis of the apparent-length values of the test lines confirmed this observation: A test line of $13 \mathrm{~mm}$ length appeared $11.23 \mathrm{~mm}$ long when it was adjoined by acuteangle context arrows, but $15.74 \mathrm{~mm}$ long when adjoined by obtuse-angle context arrows. A test line of $26 \mathrm{~mm}$ length appeared $23.75 \mathrm{~mm}$ long when adjoined by acute-angle context arrows, but $29.54 \mathrm{~mm}$ when adjoined by obtuse-angle context arrows. (When no context arrows were presented, the test lines appeared 12.92 and, respectively, $25.86 \mathrm{~mm}$ in length.) Table 1 summarizes the magnitudes of the mis-judgments in the various test line and context arrow conditions.

\section{Discussion}

Thus, the apparent length of the test line varied systematically, depending on the angle of the context arrows: A test line adjoined by acute-angle context arrows 
Table 1.

Apparent lengths and relative mis-judgments (in parentheses) of the test lines as a function of context arrows in Experiment 1

\begin{tabular}{lll}
\hline Context arrows & Test line $(\mathrm{mm})$ & \\
\cline { 2 - 3 } & 13 & 26 \\
\hline Control & $12.92(-0.08)$ & $25.86(-0.14)$ \\
Acute & $11.23(-1.77)$ & $23.75(-2.25)$ \\
Obtuse & $15.74(+2.74)$ & $28.54(+3.54)$ \\
\hline
\end{tabular}

appeared smaller, whereas a test line adjoined by obtuse-angle context arrows appeared longer. Furthermore, the apparent-length modulation was overall larger for the longer target lines than for the shorter distractor lines (target vs. distractor: $+3.54 v s$. $+2.74 \mathrm{~mm}$ magnification with obtuse-angle context arrows, -2.25 vs. $-1.77 \mathrm{~mm}$ de-magnification with acute-angle context arrows), consistent with the rationale of the visual-search Experiment 2.

Note that, in absolute terms, the magnitudes over the over- and under-judgements of the target and distractor lines with obtuse- and acute-angle context arrows are at variance with Nakagawa (1958). For a given angle of the context arrow fins (obtuse or acute), Nakagawa reported the magnitude of the illusion to be influenced by the length of the fins: For obtuse-angle arrows, the over-judgment was at maximum when the length of the arrow fins was one-third of the test line; for acute-angle context arrows, the test line was judged the shorter, the shorter were the fins. Applied to the stimuli used in Experiment 1, the mis-judgment should have been larger for distractor configurations (over-judgment with obtuse-angle context arrows; under-judgment acute-angle context arrows), making the apparentsize difference between target and distractors less than the retinal-size difference, in both context arrow conditions. However, in Experiment 1, with obtuse-angle context arrows, the target line was judged $3.54 \mathrm{~mm}$ longer, and distractor lines $2.74 \mathrm{~mm}$ longer. Similarly, with obtuse-angle context arrows, the target line was judged $2.25 \mathrm{~mm}$ shorter, and distractor lines $1.77 \mathrm{~mm}$ shorter.

However, when the mis-judgment is related to test line length, the pattern changes. With obtuse-angle context arrows, the relative over-judgment was slightly larger for distractor than for target configurations (1.21-fold vs. 1.14-fold over-judgment). For distractor configurations, the ratio of arrow fin to test line length was close to one-third $(5: 13=0.38)$; in contrast, the ratio was less than one third for target configurations $(5: 26=0.19)$. Thus, the relative mis-judgments are in accordance with Nakagawa (1958). With acute-angle context arrows, Nakagawa reported increasing under-judgment of test line length with decreasing length of the arrow fins. This was confirmed by Experiment 1, in relative terms. The arrow fins were comparatively shorter for target than for distractor configurations. Correspondingly, the relative under-judgment was larger for target than for distractor configurations (0.91-fold vs. 0.86-fold). 


\section{EXPERIMENT 2}

The visual search Experiment 2 introduced adapted control conditions (without context arrows) in which the retinal lengths of the test lines was matched with the apparent lengths of test lines under obtuse- and acute-angle context arrow conditions, based on the estimates obtained in Experiment 1. These control conditions were contrasted with search conditions in which obtuse- and acuteangle context arrows (Müller-Lyer configurations) were permitted to modulate the apparent length of the test lines. If target detection under visual search conditions is based on a pre-attentive representation of the test lines' apparent length, similar RT patterns were expected to be manifest under both adapted control and context arrow conditions.

\section{Method}

Participants. Eighteen observers (16 female; ages ranging from 20 to 35 years; all with normal or corrected-to-normal vision) participated in a single experimental session. All observers had experience with visual search tasks, and 14 had participated in Experiment 1.

Apparatus. Reaction times (RTs) were measured from the onset of the test stimuli. Responses were recorded from an ERTS keyboard (lower right button for target-present responses, lower left button for target-absent responses). Observers viewed the display from a distance of $60 \mathrm{~cm}$.

Stimuli. Stimulus displays (see Fig. 3) consisted of black horizontal test lines and adjoining context arrows, presented on a white background. Observers had to discern whether one of the test lines (the target) was longer than the other test lines (distractors). On half the trials, a target line was present in the display, with target position (on an imaginary circle) varying randomly across trials.

In the experimental conditions, the target line was $26 \mathrm{~mm}$ in length and the distractor lines $13 \mathrm{~mm}$. Each test stimulus was adjoined by a context arrow (head) at each end of the line. The context arrow heads were $5 \mathrm{~mm}$ in line length and either $45^{\circ}$ (acute) or $135^{\circ}$ (obtuse) in angle (angle between each fin of the context arrows and the test line). All context arrows were of equal angle (homogeneous) in a given search display. According to the results of Experiment 1, obtuse-angle context arrows increased the apparent lengths of the test lines, with a greater modulation for the longer target than for the shorter distractor lines; and acute-angle context arrows decreased the apparent lengths of the test lines, again with a greater effect for target than for distractor lines.

In the adapted control conditions, only the target and distractor test lines were presented (i.e. there were no context arrows). Based on the apparent-length estimates derived in Experiment 1, in the control conditions, the (retinal) length of the distractor lines was set to 11.25 and $15.75 \mathrm{~mm}$, respectively, matching 


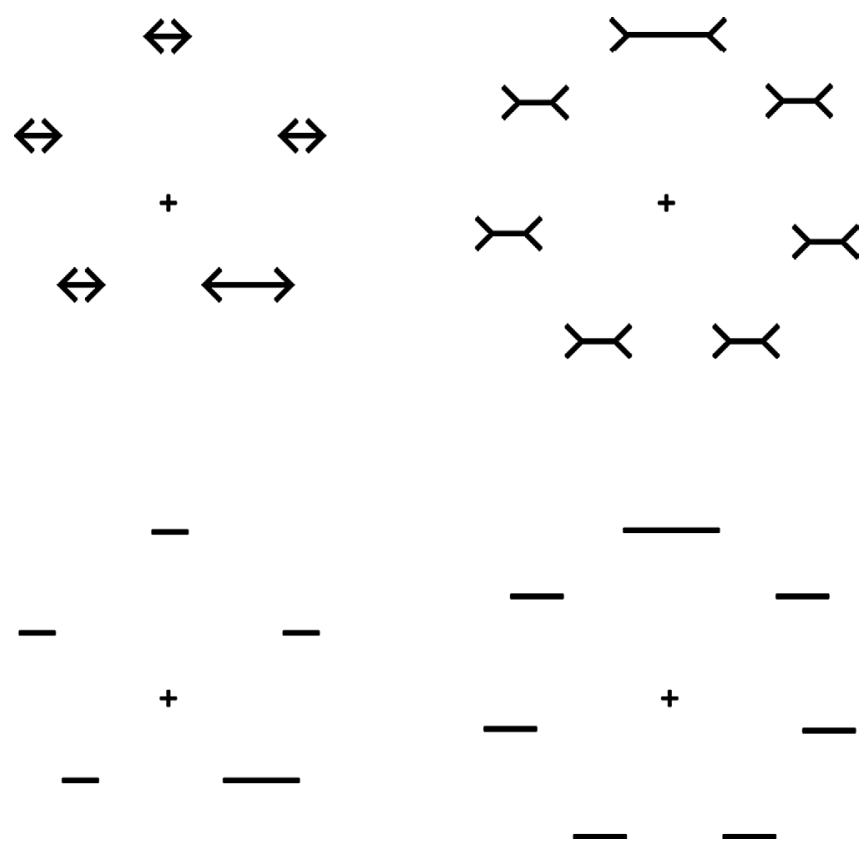

Figure 3. Example displays, with target present, for acute- and obtuse-angle experimental and the corresponding control conditions in Experiment 2, for 5- and 7-element displays, respectively.

the respective apparent lengths of distractor stimuli with acute- and obtuse-angled context arrows; and the length of the target lines was set to 23.75 and $29.50 \mathrm{~mm}$, respectively, matching the respective apparent lengths of target stimuli with acuteand obtuse-angle context arrows.

Each display consisted of a central fixation cross and three, five, or seven test lines with (experimental conditions) or without (control conditions) context arrows, respectively. The stimuli were positioned on an imaginary circle (with a radius of $70 \mathrm{~mm}$ ) around the center of the screen, with equal separation between the configurations. (The number of configurations was limited to seven, in order to permit a direct comparison with the experiments of Busch and Müller.) The overall display dimensions were $20^{\circ}$ of visual angle in height by $20^{\circ}$ in width.

Design and procedure. The independent variables in Experiment 2 were: display size $(3,5$, or 7 elements), target (present, absent), test condition (control, experimental), and context condition (acute-, obtuse-angle arrows, or corresponding control conditions without context arrows).

The experiment consisted of 12 trial blocks, each of 60 test trials. Display size and test conditions were kept constant throughout each block, but varied across blocks, with order of blocks counterbalanced across observers. Context (arrow) condition was varied randomly within trial blocks in order to force observers to base their target-present/absent responses on the length difference between the target and distractor test lines, rather than the total length of the Müller-Lyer configurations. 
That is, randomization was intended to ensure that observers 'focus' processing on the test lines and use a single 'criterion length difference' for ascertaining target presence in all conditions. In total, the experiment consisted of 720 test trials (30 trials for each of the 24 display size $\times$ target $\times$ test $\times$ context conditions) and lasted approximately 30 minutes.

Each block started with 4 unrecorded practice trials (one trial for each of the four different trial types within the block). Observers initiated a block by pressing one of the response buttons. Each trial began with the presentation of a small black cross in the center of the screen, which observers were instructed to fixate. The display elements appeared $750 \mathrm{~ms}$ later around this fixation cross. The display elements remained visible until the observer gave a button press response. Following a correct response, the screen went blank (white) for an inter-trial interval of $750 \mathrm{~ms}$. After in incorrect response, the observer received an acoustic signal ('beep') for $200 \mathrm{~ms}$ and the inter-trial interval was increased to $1750 \mathrm{~ms}$. Then the next trial began, unless the end of a block was reached. Observers were instructed to respond 'target present' or 'target absent' as fast and as accurately as possible.

\section{Results}

$R T$ analysis. Prior to analysis, for each observer, the mean RTs and associated standard deviations were calculated for each condition. Next, RTs outside the range \pm 2.5 standard deviations from the mean were eliminated as 'outliers' before recalculating the mean RTs for further data analysis. This procedure produced a loss of $2.6 \%$ of the data. Figure 4 presents the group mean correct target-present and target-absent RTs as a function of display size, separately for the control and experimental conditions. Table 2 presents the mean correct RTs, the mean base RTs (y-intercepts) and search rates (slopes) of the RT/display size functions (all with associated standard errors), and the mean error rates and error RTs.

First, observers' mean correct RTs were examined by an overall ANOVA, with target (present, absent), display size $(3,5,7)$, test condition (control, experimental), and context condition ('acute-angle', 'obtuse-angle') as factors. This ANOVA revealed the main effects of target $(F(1,17)=26.003, p<0.001)$ and context condition $(F(1,17)=58.820, p<0.001)$ and the target $\times$ context condition interaction to be significant $(F(1,17)=153.073, p<0.001)$. Target-present RTs were overall faster than absent RTs (435 vs. $470 \mathrm{~ms}$ ), and, combined across target-present and absent responses, RTs were faster in 'acute-angle' relative to 'obtuse-angle' conditions (438 vs. 467 ms). However, on target-present trials, RTs were faster in 'obtuse-angle' relative to 'acute-angle' conditions (431 vs. $439 \mathrm{~ms}$ ), whereas target-absent RTs displayed the reverse pattern (503 and $438 \mathrm{~ms}$ for 'obtuse-angle' and 'acute-angle' conditions, respectively).

The interaction between target and context condition was examined further by separate ANOVAs for target-present and absent RTs. For target-present RTs, the ANOVA revealed the main effects of context condition $(F(1,17)=9.630$, $p=0.006)$ and display size $(F(2,34)=4.775, p=0.024)$ to be significant. 
Target Present

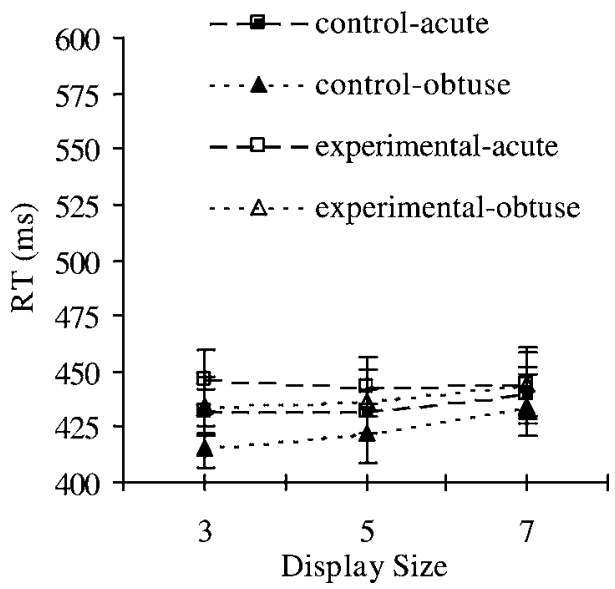

(A)
Target Absent

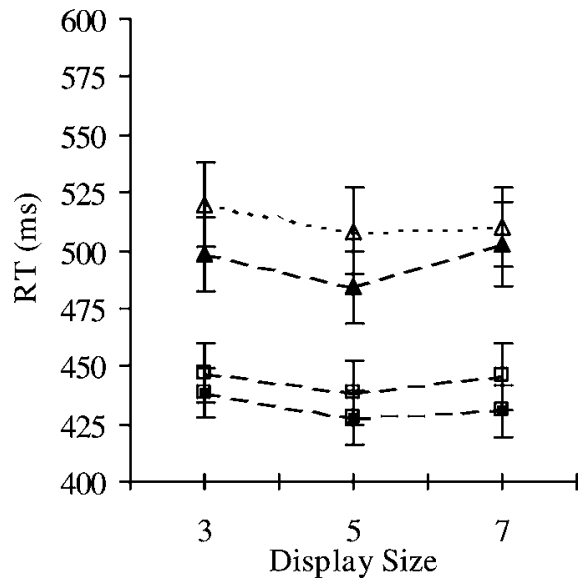

(B)

Figure 4. Mean correct RTs, with associated standard errors (bars), in Experiment 2, separately for target-present (4A) and -absent trials (4B). Acute- and obtuse-angle context arrow conditions are represented by dashed and dotted lines, respectively. Experimental and control conditions are represented by open and filled, respectively.

\section{Table 2.}

Mean correct reaction times, base reaction times, search rates (with associated standard errors), error rates, and mean error reaction times in Experiment 2. Reaction times and error rates combined across display size conditions

\begin{tabular}{|c|c|c|c|c|}
\hline Context condition & Control-Acute & Control-Obtuse & Experimental-Acute & Experimental-Obtuse \\
\hline \multicolumn{5}{|l|}{ Target present } \\
\hline Mean correct RTs (ms) & $434[9.86]$ & 424 [11.39] & $444[13.38]$ & $438[14.48]$ \\
\hline Base RTs (ms) & $423[5.31]$ & 399 [3.79] & $446[3.03]$ & $426[3.79]$ \\
\hline Search rates (ms/item) & $2.25[1.01]$ & $4.75[0.72]$ & $-0.50[0.57]$ & $2.25[0.72]$ \\
\hline Error rates $(\%)$ & 5.07 & 4.20 & 5.44 & 4.33 \\
\hline Mean error RTs (ms) & 410 & 433 & 424 & 463 \\
\hline \multicolumn{5}{|l|}{ Target absent } \\
\hline Mean correct RTs (ms) & $432[10.31]$ & $494[15.56]$ & 443 [11.96] & $512[16.92]$ \\
\hline Base RTs (ms) & $441[10.62]$ & 489 [24.29] & $455[33.40]$ & $523[9.87]$ \\
\hline Search rates (ms/item) & $-2.00[2.02]$ & $1.00[4.61]$ & $-0.50[6.35]$ & $-2.25[1.87]$ \\
\hline Error rates $(\%)$ & 0.81 & 5.56 & 0.81 & 4.76 \\
\hline Mean error RTs (ms) & 565 & 433 & 476 & 450 \\
\hline
\end{tabular}

Though numerically different, target-present RTs did not differ reliably between the two test conditions $(F(1,17)=2.465, p=0.135 ; 429$ and $441 \mathrm{~ms}$ for control and experimental conditions, respectively). However, context condition had a significant effect on search performance: RTs were slightly, but reliably faster when test lines were adjoined by obtuse-angle arrows and in the corresponding control condition 
(431 ms) than when test lines were adjoined by acute-angle arrows and in the corresponding control condition (439 ms). Target-present RTs also exhibited a significant increase with increasing display size. However, the increase was only about 2 ms/element (431, 433, and $440 \mathrm{~ms}$ with 3-, 5-, and 7-element displays, respectively), that is, well within the range taken to be indicative of efficient, parallel search $(<10 \mathrm{~ms} /$ element; see below for a more detailed analysis of search rates).

For target-absent RTs, the ANOVA revealed responses to be significantly slower when the test lines were adjoined by obtuse-angle context arrows and in the corresponding control condition than when test lines were adjoined by acute-angle context arrows and in the corresponding control condition $(503 v s .438 \mathrm{~ms}$; main effect of context condition $(F(1,17)=9.630, p=0.006)$; this pattern is the reverse of that observed with target-present RTs. The main effect of test condition was not significant $(F(1,17)=2.714, p=0.118 ; 463$ and $478 \mathrm{~ms}$ for control and experimental conditions, respectively), as with target-present RTs.

Two further ANOVAs, with main terms for target, test condition, and context condition, examined the base RTs (y-intercepts) and search rates (slopes) of the RT/display size functions (see Table 2). The base RT effects mirrored those of the mean RTs. Base RTs were faster for target-present relative to absent trials (423 vs. $477 \mathrm{~ms} ; F(1,17)=25.971, p<0.001)$ and faster for 'acute-angle' relative to 'obtuse-angle' conditions (441 vs. $459 \mathrm{~ms} ; F(1,17)=24.062, p<0.001$ ). Further, on target-present trials, base RTs were faster in 'obtuse-angle' relative to 'acute-angle' conditions (413 vs. $434 \mathrm{~ms}$ ); this pattern was reversed on target-absent trials $(506 v s$. $448 \mathrm{~ms}$; target $\times$ context condition interaction: $F(1,17)=29.872$, $p<0.001$ ). Test condition had no significant influence on base RTs (main effect: $F(1,17)=3.887, p=0.065)$. The search rates did not differ significantly between the experimental conditions $(F(1,17)=3.442, p=0.081$, for target; $F(1,17)=0.501, p=0.489$, for test condition; $F(1,17)=2.601, p=0.125$, for context condition).

Error analysis. The error data (see Table 2) were analyzed by an ANOVA with display size, target, test condition, and context condition as factors. This analysis revealed significant main effects of target $(F(1,17)=14.702, p=0.001)$ and context condition $(F(1,17)=25.911, p<0.001)$, and a significant interaction between these two factors $(F(1,17)=18.935, p<0.001)$. Observers made more target miss than false-alarm errors $(4.76 \%$ vs. $2.98 \%)$. Fewer errors were made with test lines adjoined by arrows of acute angle and in the corresponding control condition $(3.03 \%)$ than with test lines adjoined by arrows of obtuse angle and in the corresponding control condition $(4.71 \%)$. Target miss and false-alarm rates were comparably high in the obtuse-angle context arrow and corresponding control conditions (4.27\% vs. 5.16\%); in contrast, in the acute-angle context arrow and corresponding control conditions, while target miss rates remained high, falsealarm rates were reduced $(5.16 \% \mathrm{vs} .0 .81 \%)$. As with the RT data, test condition had no influence on error rates (main effect: $F(1,17)=0.053, p=0.821$ ). 


\section{Discussion}

The results of Experiment 2 show that the search was performed efficiently in all conditions. Even though target-present RTs increased significantly with increasing display size, the slopes of the positive RT/display size functions were well below $10 \mathrm{~ms} /$ item. This is widely accepted to indicate efficient search performance (Treisman and Gelade, 1980; Wolfe, 1998).

Furthermore, search RTs were influenced by the angle of the context arrows. Target-present responses were significantly faster when test lines were adjoined by obtuse-angle arrows and in the corresponding control condition than when test lines were adjoined by acute-angle arrows and the corresponding control condition. This context effect was independent of display size, indicating parallel processing of the (retinal plus) apparent lengths of the test lines in the experimental and of the retinal lengths in the control conditions.

Experiment 2 was designed to compare the overall (target-present) RT performance between the adapted-control and experimental conditions. Importantly, statistical analyses failed to find a significant influence of the test condition (control vs. experimental) on the RT (and error) data. This supports three conclusions:

(1) The differential apparent-length modulation between the target and distractors test lines in the experimental conditions produced equivalent effects to the differential retinal-length variation in the control conditions. Given that search was performed efficiently, spatially in parallel in both control and experimental conditions, one can conclude that, in the latter conditions, apparent-line length was computed, and compared, in parallel across the Müller-Lyer configurations in the display, in the same way as retinal length in the control conditions (see Note 2).

(2) There were no special (additive) costs in the experimental relative to the control conditions that could have arisen from additional processing of the taskirrelevant context arrows (though there were numerical costs, of $12 \mathrm{~ms}$, for the experimental conditions). This is contrary to Busch and Müller (2004), who found a significant (additive) cost between control and experimental conditions. Busch and Müller took this cost to reflect the additional processing of the task-irrelevant context circles in the Ebbinghaus configurations, which (besides modulating the test circles' apparent size) cause interference in visual search and, therefore, need to be suppressed (see Müller and Busch, 2003). The lack of such a cost with Müller-Lyer configurations suggests that, although they are processed configurally (thereby producing apparent-length effects; see conclusion (1) above and $c f$. Rensink and Enns, 1995), task-relevant line components can be efficiently accessed and compared in parallel without significant interference from the arrow fins. The interference is minimal, possibly because the context arrows can be efficiently de-selected, due to their featural dissimilarity relative to the test lines (oblique arrow fins $v s$. horizontal test lines; $c f$. Duncan and Humphreys, 1989). 
(3) The specific pattern of effects on target-present trials — advantage for obtuseangle and corresponding control conditions over acute-angle and corresponding control conditions - supports the view that targets achieve 'saliency' and are detected based on the parallel computation of (apparent or retinal) length differences between the target and distractor test lines, rather than their ratio. Recall that, while the length difference was larger under obtuse-angle and corresponding control conditions relative to acute-angle and corresponding control conditions $(13.75 \mathrm{~mm}$ vs. $12.50 \mathrm{~mm})$, the target-to-distractor ratio was lower (1.87 vs. 2.11; see Note 1$)$. Therefore, if the ratio were critical for determining search performance, there should have been a disadvantage (rather than the observed advantage) for obtuse-angle and corresponding control conditions relative to acute-angle and corresponding control conditions.

However, target-absent RTs exhibited the reverse pattern to target-present RTs: they were slow when (distractor) test lines were adjoined by obtuse-angled arrows and in the corresponding control condition, and fast when they were adjoined by acute-angled arrows and in the corresponding control condition. Although this pattern would be consistent with (target-absent) response thresholds being set in accordance with the length ratio of the target to the distractor test lines (Adrian von Mühlenen, personal communication, August 28, 2003), the 'ratio' account fails to explain why the target-present responses exhibited an RT advantage (rather than a disadvantage) for obtuse-angled arrow and corresponding control conditions relative to acute-angled arrow and corresponding control conditions. While it is conceivable that observers apply separate threshold setting 'rules' for responding target present and absent (target-distractor difference vs. target-to-distractor ratio), most models of (efficient) visual search assume that target-absent reactions are a default response given when no target was detected within a set period of time (i.e. the absent-response threshold is determined by the distribution of the time it takes to discern the presence of a target; e.g. Chun and Wolfe, 1996).

Extending this line of reasoning, the differential pattern of target-present and absent RT effects may be understood in terms of a random-walk RT model (e.g. Ratcliff et al., 1999) in which target-present and absent response thresholds (or, alternatively, the evidence in favor of a positive $v s$. a negative decision) are set with reference to the perceived length of all the items in the display. In the acutearrow and the corresponding control condition, on target-absent trials, all test lines appeared or were shorter (relative to the obtuse-arrow and the corresponding control condition), thereby biasing observers to respond 'target-absent'. In contrast, in the obtuse-arrow and the corresponding control condition, all test lines appeared or were longer, thereby biasing observers away from responding 'target-absent'; then, since all stimuli appeared large, it would have taken a long time of further 'sampling' to reach a negative decision. This interpretation is supported by the error analysis: False-alarm rates were lower when test lines were adjoined by acuteangle rather than obtuse-angle arrows $(0.81 \%$ vs. $4.76 \%$; corresponding control 
conditions were $0.81 \%$ vs. $5.56 \%$ ), while there was little difference in terms of miss rates (5.44\% vs. $4.33 \%$; control conditions $4.20 \%$ vs. 5.07\% — see Note 3).

The above interpretation rests on the assumption that special biasing processes (based on estimates of overall stimulus length) that determine negative decisions on target-absent trials may not come into play on target-present trials, when the rapid computation of a length contrast signal between the target and distractor lines shifts the system towards a positive decision in a single step. However, although the error rate effects are consistent with this assumption, it cannot be ruled out that the biasing processes operate on target-present trials, too (in terms of a speedaccuracy tradeoff, SATO). Therefore, to examine for a SATO, the error RTs were calculated and compared with the correct RTs (see Table 2). This comparison revealed that, with obtuse-angle arrows and in the corresponding control condition, the mean false-alarm RTs were not faster (but, if anything, slower) than the mean hit RTs: $450 \mathrm{~ms} v s .438 \mathrm{~ms}$ and $433 \mathrm{~ms} v s .424 \mathrm{~ms}$, respectively (the corresponding values were $476 v s$. $444 \mathrm{~ms}$ and 565 vs. $434 \mathrm{~ms}$ for the acute-angle arrow and the corresponding control condition, respectively). Thus, there is no evidence to suggest confounding of the pattern of RT effects by a SATO.

\section{EXPERIMENT 3}

Nevertheless, another experiment (Experiment 3) was carried out that was devised to avoid confounding of the effects of the Müller-Lyer illusion on target detection RTs by a SATO 'by design'. In Experiment 3, all three types of stimuli, test lines without context arrows, with obtuse-angle arrows, and with acute-angle arrows, were presented in equal numbers in a single display (i.e. the stimuli were heterogeneous), and any type of stimulus could be or, respectively, contain the (longer) target test line on a given trial. In this situation, the target-absent decision criterion would be expected to be set in accordance with the longest times it takes to make a target-present decision (Chun and Wolfe, 1996), and no special biasing processes (as outlined in the Discussion of Experiment 2) ought to be involved on target-present trials.

In addition to avoiding potentially confounding SATOs, Experiment 3 was designed to address another problem not conclusively resolved by Experiment 2: similar to earlier studies (e.g. Aks and Enns, 1996; Found and Müller, 2001; Humphreys et al., 1994), the evidence for parallel coding of the Müller-Lyer apparent-size illusion provided by Experiment 2 rests on base RT (intercept), rather than search rate (slope), effects. (Slope effects were not expected given that the search rates were near-zero ms/item under control conditions and, thus, could not be improved by the operation of the Müller-Lyer apparent-size illusion.) The intercept effects leave the possibility that observers detected the target test line on the basis of its retinal size difference relative to the distractor test lines and that they were deriving apparent-size information, modulating their RTs, only after having deployed focal attention to the target. Thus, Experiment 2 does not 
conclusively answer whether apparent size is computed separately for each MüllerLyer configuration in the display.

To address this issue, in Experiment 3, observers were presented with displays of heterogeneous stimuli, making it harder for them to base their search solely on retinal-size comparisons of the test circles (because it would be harder to de-select heterogeneous context circles; see Duncan and Humphreys, 1989). If, nevertheless, similar results were obtained as in (the homogeneous context arrow conditions of) Experiment 2, this would strengthen the case for the suggestion that apparent size is computed separately for each test line.

\section{Method}

Participants. Eighteen observers (15 female; ages ranging from 21 to 50 years) participated in a single experimental session.

Stimuli. The stimuli were similar to those used in Experiment 2. However, the display elements were 'heterogeneous' in that they consisted, in equal numbers, of test lines with acute- and with obtuse-angle context arrows as well as test lines without context arrows. For displays to contain equal numbers of each type of test line, display size was increased to three, six, or, respectively, nine test lines, with the various types of lines (Müller-Lyer configurations) allocated, in random order, to equidistant positions on an imaginary circle around the screen center (see Fig. 5 for example displays). Thus, with display size 3 for example, there was one test line with acute-angle context arrows, one with obtuse-angle arrows, and one without arrows. Each type of test line was equiprobable to be the (longer) target line. With larger display sizes, on target-present trials, displays contained at least one distractor configuration of the same type as the target configuration.

Design and procedure. The independent variables in Experiment 3 were: display size (3, 6, or 9 elements), target (present or absent), and target context arrows (zero, acute-, or obtuse-angle). Context-arrow conditions refer to the angle of context arrows adjoining the target line.

Experimental sessions consisted of 540 trials ( 30 trials for each of the 18 display size $\times$ target $\times$ context-arrow conditions) and lasted approximately 20 minutes.

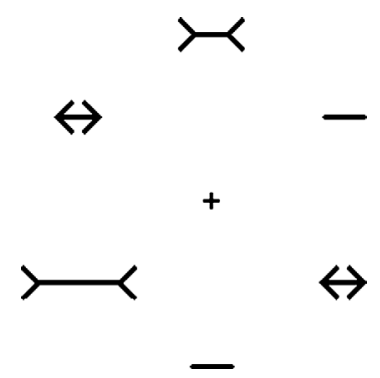

Figure 5. Example display used in Experiment 3. 
The session was subdivided into 9 blocks of 6 practice plus 60 test trials. Display size was kept constant throughout each block, but varied across blocks. The order of display size blocks was counterbalanced across observers. Target present/absent and context-arrow conditions were varied randomly within each block.

\section{Results}

RT analysis. Prior to analysis, outlier RTs $(2.7 \%)$ were eliminated from the data. Figure 6 presents the group mean correct target-present and target-absent RTs as a function of display size; Table 3 summarizes the corresponding mean correct RTs, base RTs, search rates, error rates, and mean error RTs.

Observers' mean correct target-present and target-absent RTs were examined by separate ANOVAs. The ANOVA of the target-present RTs, with the factors display size (3, 6, 9 elements) and target context arrows (zero, acute-, obtuse-angle), revealed only a significant main effect of target context arrows $(F(2,34)=25.553$, $p<0.001)$. Target-present RTs did not vary significantly as a function of display size $(608,617$, and $631 \mathrm{~ms}$ for 3, 6, and 9-element displays, respectively; main effect: $F(2,34)=0.525, p=0.601)$. However, the angle of the context arrows adjoining the target had a large effect on search performance: RTs were fastest when the target line was adjoined by context arrows of obtuse angle $(555 \mathrm{~ms})$, intermediate in the control condition in which the target line had no context arrows $(591 \mathrm{~ms})$, and slowest with target context arrows of acute angle $(710 \mathrm{~ms})$. This pattern of effects was not significantly influenced by display size (target context arrows $\times$ display size interaction: $F(4,68)=1.461, p=0.266)$. Paired $t$-tests among the three target context arrow conditions (data combined across display

Target Present and Absent

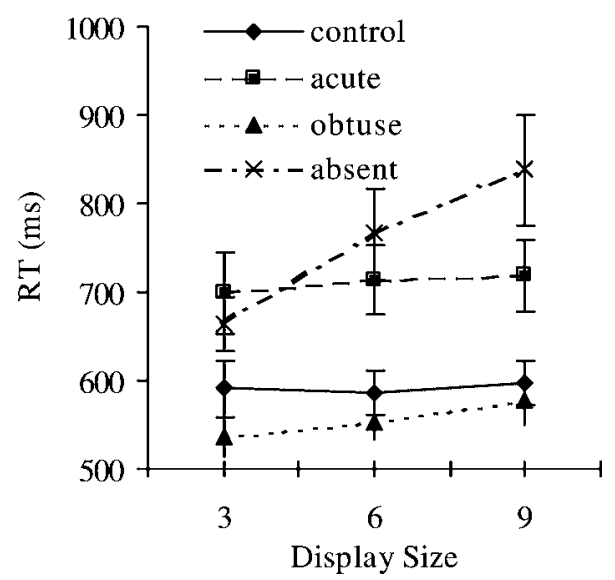

Figure 6. Mean correct RTs, with associated standard error (bars), in Experiment 3, separately for target-present and target-absent trials. Target-present control, acute-angle, and obtuse-angle context arrow conditions are represented by solid, dashed, and dotted lines respectively. Target-absent RTs are represented by a dashed-dotted line. 
size) revealed all differences to be significant (control vs. acute-angle arrows: $t(17)=-6.651, p<0.001$; control vs. acute-angle arrows: $t(17)=3.919$, $p=0.001$; acute-angle $v s$. obtuse-angle arrows: $t(17)=7.369, p<0.001)$.

Two further ANOVAs, with target context arrows (control, acute-angle, obtuseangle) as factor, were conducted to examine search rate and base RT data. Search rates did not differ significantly among the context conditions (1.17, 3.33, $6.67 \mathrm{~ms} /$ item for control, acute-angle, and obtuse-angle context arrows; $F(2,34)=$ $1.541, p=0.244)$. However, base RTs were fastest with obtuse-angle context arrows $(515 \mathrm{~ms})$, intermediate in the control condition $(584 \mathrm{~ms})$, and slowest with acute-angle context arrows $(689 \mathrm{~ms} ; F(2,34)=9.354, p=0.002)$.

A one-factorial ANOVA of the target-absent RTs revealed a significant display size effect $(F(2,34)=9.616, p=0.002)$ : RTs were 664,766 , and $837 \mathrm{~ms}$ for 3 , 6 , and 9-element displays, respectively. (The search rate was $28.83 \mathrm{~ms} /$ item and the base RT $582 \mathrm{~ms}$.)

Error analysis. Error rates and associated error RTs are presented in Table 3. Analogously to the target-present and target-absent RTs, the target-miss and falsealarm data were analyzed in separate ANOVAs. The ANOVA of the target-miss errors revealed only a significant main effect of target context arrows $(F(2,34)=$ 16.816, $p<0.001$; display size main effect: $F(2,34)=0.518, p=0.606$; interaction: $F(4,68)=0.791, p=0.550)$. Targets were likely to be missed when the target line was adjoined by acute-angle arrows (9.39\%); but miss rates were quite low for targets without context arrows $(2.60 \%)$ or with arrows of obtuse angle $(1.80 \%)$. [The ANOVA of the false-alarm errors revealed no significant effect of display size $(F(2,34)=1.149, p=0.342)$.] Thus, the pattern of target miss errors

Table 3.

Mean correct reaction times, base reaction times, search rates (with associated standard errors), error rates, and mean error reaction times in Experiment 3. Reaction times and error rates combined across display size conditions

\begin{tabular}{llll}
\hline Context condition & Control & Acute & Obtuse \\
\hline Target present & & & \\
Mean correct RTs (ms) & $591[25.95]$ & $710[37.83]$ & $555[21.59]$ \\
Base RTs (ms) & $584[9.35]$ & $689[6.23]$ & $515[6.23]$ \\
Search rates (ms/item) & $1.17[1.44]$ & $3.33[0.96]$ & $6.67[0.96]$ \\
Error rates $(\%)$ & 2.60 & 9.39 & 1.80 \\
Mean error RTs (ms) & 648 & 720 & 653 \\
Target absent & & & \\
Mean correct RTs (ms) & & $756[45.25]$ & \\
Base RTs (ms) & & $582[19.33]$ & \\
Search rates (ms/item) & & $28.83[2.98]$ & \\
Error rates $(\%)$ & & 858 & \\
Mean error RTs $(\mathrm{ms})$ & & & \\
\hline
\end{tabular}


are in the same direction as the RT effects, arguing against a confounding of the RT effects by a SATO.

\section{Discussion}

The results of Experiment 3 again show that targets were searched efficiently, in parallel across the display (non-significant display size effect on target-present RTs). In Experiment 3, observers took overall longer to discern the presence and, particularly, absence of a target relative to Experiment 2. This difference is likely due to the more difficult task in Experiment 3, which required search through heterogeneous, as compared with homogeneous, display (distractor) elements ( $c f$. Duncan and Humphreys, 1989).

Despite the increased task difficulty, however, the results of Experiment 3 demonstrate a significant influence of the angle of target context arrows on search performance: Targets were detected faster relative to the control condition when the target line was adjoined by obtuse-angle arrows, increasing the target's apparent size (by the operation of the Müller-Lyer illusion) and thereby its length difference relative to the distractors. In contrast, targets were detected slower relative to the control condition when the target line was adjoined by acute-angle arrows, decreasing the target's apparent size and thereby its length difference relative to the distractors. This pattern of effects, which was independent of display size, replicates Busch and Müller's (2004) findings with the Ebbinghaus illusion and confirms that the apparent size of display objects is derived and compared at an early, pre-attentive stage of visual processing.

One objection that may be leveled against the results of Experiment 3 is that the increased RT differences between the various context arrow conditions reflect serial processing of three types of display elements: Configurations with obtuseangle arrows are searched first for the presence of a target (because they are overall largest), next elements without context arrows, and finally configurations with acute-angle arrows. Since the different types of element are searched and compared in parallel, the result is an additive RT effect of context arrow condition (that is independent of display size). However, this account fails to explain why the effect was manifest with three-element displays (that contained one element of each type), in which case target presence could only be ascertained by comparisons across the various types of element. Second, it fails to explain why elements with acute-angle arrows would have a lower priority of processing than elements without context arrows. An account in terms of 'saliency' fails because, arguably, acute-angle configurations are more salient than test lines without context arrows. One possibility that remains is that acute-angle configurations were processed last because they 'appeared' shortest (and obtuse-angle configurations first because they 'appeared' longest). However, if this were true, the account would have to admit a role of the Müller-Lyer apparent-length modulation in (pre-attentively) determining the order with which the various types of display elements were processed. Thus, in 
summary, it seems difficult to account for the data of Experiment 3 without recourse to a spatially parallel, pre-attentive effect of the Müller-Lyer illusion.

\section{GENERAL DISCUSSION}

The present findings provide further evidence that observers are able to search for a size-defined target efficiently. In all search experiments, the slopes of the targetpresent RT/display size functions (as well as the slopes of almost all target-absent functions) were below $10 \mathrm{~ms} /$ item. This was the case not only in Experiment 2, in which displays contained only a single type of element (homogeneous displays), but also in Experiment 3, in which displays contained all three types of elements (heterogeneous displays).

Most importantly, the present findings support and extend the conclusion (e.g. Busch and Müller, 2004) that apparent object size is computed and represented pre-attentively. In particular, using Müller-Lyer configurations, visual search performance was revealed to be systematically influenced by the context arrows adjoining the test lines in the displays. Obtuse-angle context arrows expedited target detection, acute-angle context arrows slowed it. This modulation was most marked in Experiment 3, which required prolonged processing to detect the target (due to the heterogeneous displays). Nevertheless, the effect of the Müller-Lyer apparentlength illusion was independent of display size (there was no effect on display size on target-present RTs), suggesting that the modulation is computed and represented pre-attentively, spatially in parallel across the display.

Experiment 2 confirmed that, with Müller-Lyer configurations, no, or only little, additional 'effort' is required to direct search processes to the test lines. This result contrasts with Busch and Müller (2004), who used the Ebbinghaus illusion to modulate apparent object size. They found qualitatively similar RT patterns, in search for size-defined targets, under adapted-control conditions (without Ebbinghaus illusion) and conditions of equivalent apparent-size modulation by the Ebbinghaus illusion. However, the latter conditions exhibited an RT cost of about $40 \mathrm{~ms}$, which was taken to reflect additional processes necessary to deal with the interference caused by the context circles (see also Müller and Busch, 2003). In contrast, with visual search through line stimuli modulated by the Müller-Lyer illusion, task-relevant (test) lines can be efficiently accessed and compared in parallel without significant interference from the (context) arrow fins. The interference is minimal, possibly because the context arrows can be efficiently de-selected, due to their featural dissimilarity relative to the test lines (oblique arrow fins $v s$. horizontal test lines). Nevertheless, the Müller-Lyer stimuli are processed configurally, as evidenced by the systematic apparent-length modulations of the test lines by the context arrows. This finding adds further support to the main conclusion drawn by Rensink and Enns (1995).

Note, though, that the present findings go significantly beyond those of Rensink and Enns. They could not definitely rule out that, in their experiments, search was based on overall configural differences, because the context arrows of the 
target configuration were always different from those of the distractor configurations (obtuse-angle vs. acute-angle arrows, or vice versa). Thus, observers could operate a strategy of searching for a differently 'angled' (rather than a differently 'sized') target object. Such a strategy was not available to observers in the present search experiments, because the target test line was not adjoined by a unique type of context arrows.

In summary, the present findings add to the increasing body of evidence that the 'basic features' - in the present study: object size — computed and represented at the pre-attentive level of processing are rather more complex than the simple visual features coded 'bottom-up' in early visual processing areas. Exactly how this is achieved needs to be clarified in further work. Presumably, however, this involves the operation of both parallel 'bottom-up' and 'top-down' (in terms of von Helmholtz's, 1896, 'inferential') processes (see Wolfe, 2003).

\section{Acknowledgements}

The authors wish to thank the reviewers for their helpful suggestions on an earlies version of the manuscript. This research was supported by a grant from the Deutsche Forschungsgemeinschaft MU 773/2-3.

\section{NOTES}

1. The opposite predictions would be made if visual-search performance were based not on the apparent-length difference of the target to the distractor test lines, but on their apparent length ratio. For the obtuse-angle context arrow condition, relative to test lines without context arrows (target-distractor difference $13.00 \mathrm{~mm}$; target-todistractor ratio of $2 / 1)$, the difference is larger $(13.80 \mathrm{~mm})$, but the ratio is smaller (1.88/1). Conversely, for the acute-angle context arrow condition, the difference is smaller $(12.52 \mathrm{~mm})$, but the ratio is larger (2.11/1). Thus, if the target-to-distractor apparent-length ratio were determining search performance, target detection should be impeded in the obtuse-angle context arrow condition, but expedited in the acuteangle condition. Note that most accounts of efficient ('pop-out') visual search, such as Wolfe's $(1994,1998)$ Guided Search theory, explicitly assume that the 'feature' difference is critical for determining search performance: Pop-out is thought to be based on 'saliency' signals that reflect the overall feature difference between the target and distractors (see also Nothdurft, 1992, 1993; Koch and Ullman, 1985).

2. This conclusion is supported by another experiment, not reported in the present study, which compared performance in experimental conditions of test lines adjoined by obtuse- and, respectively, acute-angle context arrows (stimuli identical to those used in Experiment 2) with a control condition in which the test lines were presented without context arrows, but had the same absolute lengths as in the experimental conditions (26 [target] and $13 \mathrm{~mm}$ [distractors]). The results showed that, while targets were detected efficiently, in parallel in all conditions (search rates 
$<10 \mathrm{~ms} /$ item), detection RTs were expedited for the obtuse-angle context arrow condition relative to the control condition ( $447 \mathrm{~ms} v s$. $451 \mathrm{~ms}$ ), but impeded for the acute-angle context arrowcondition relative to the control condition (mean RTs: $456 \mathrm{~ms} v s .451 \mathrm{~ms}$ ). This pattern is as expected assuming that obtuse-angle context arrows increase the apparent-length difference between the target and distractor test lines, while acute-angle context arrows decrease it.

\section{REFERENCES}

Aks, D. J. and Enns, J. T. (1992). Visual search for direction of shading is influenced by apparent depth, Perception and Psychophysics 52, 63-74.

Aks, D. J. and Enns, J. T. (1996). Visual search for size is influenced by a background texture gradient, J. Exper. Psychol.: Human Perception and Performance 22, 1467-1481.

Bilsky, A. B. and Wolfe, J. M. (1995). Part-whole information is useful in visual search for size $\times$ size but not orientation $\times$ orientation conjunctions, Perception and Psychophysics 57.749-760.

Busch, A. and Müller, H. J. (2004). The Ebbinghaus illusion modulates visual search for sizedefined targets: Evidence for pre-attentive processing of apparent object size, Perception and Psychophysics (in press).

Chun, M. and Wolfe, J. M. (1996). Just say no: How are visual searches terminated when there is no target present? Cognitive Psychology 30, 39-78.

Day, R. H. (1989). Natural and artificial cues, perceptual compromise and the basis of veridical and illusory perception, in: Human Information Processing: Measures and Mechanisms, Vickers, D. and Smith, D. L. (Eds), pp. 107-129. Elsevier Science, North Holland, The Netherlands.

Day, R. H. (1990). The Bourdon illusion in haptic space, Perception and Psychophysics 47, 400-404.

DeLucia, P. and Hochberg, J. (1985). Illusions in the real world and in the mind's eye [Abstract], Proc. Eastern Psychol. Assocn 56, 38.

DeLucia, P. and Hochberg, J. (1986). Real-world geometrical illusions: Theoretical and practical implications [Abstract], Proc. Eastern Psychol. Assocn 57, 63.

DeLucia, P. and Hochberg, J. (1991). Geometrical illusions in solid objects under ordinary viewing conditions, Perception and Psychophysics 50, 547-554.

Duncan, J. and Humphreys, G. W. (1989). Visual search and stimulus similarity, Psych. Rev. 96, 433458.

Duncan, J. and Humphreys, G. W. (1992). Beyond the search surface: Visual search and attentional engagement, $\underline{\text { J. Exper. Psychol.: Human Perception and Performance 18. 578-588. }}$

Ebbinghaus, H. (1913). Grundzüge der Psychologie. Veit, Leipzig.

Enns, J. T. (1992). Sensitivity of early human vision to 3-D orientation in line-drawings, Canad. J. Psychol. 46, 143-169.

Enns, J. T. and Rensink, R. A. (1990a). Influence of scene-based properties on visual search, $\underline{\text { Science }}$ 247, 721-423.

Enns, J. T. and Rensink, R. A. (1990b). Sensitivity to three-dimensional orientation in visual search, Psychol. Sci. 1, 323-326.

Enns, J. T and Rensink, R. A. (1991). Preattentive recovery of three-dimensional orientation from line drawings, Psychol. Rev. 98, 335-351.

Epstein, W. and Babler, T. (1990). In search of depth, Perception and Psychophysics 48, 68-76.

Epstein, W., Babler, T. and Bownds, S. (1992). Attentional demands of processing shape in threedimensional space: Evidence from visual search and precuing paradigms, J. Exper. Psychol.: Human Perception and Performance 18, 503-511.

Epstein, W. and Broota, K. D. (1986). Automatic and attentional components in perception of size-ata-distance, Perception and Psychophysics 40, 256-262. 
Found, A. and Müller, H. J. (2001). Efficient search for size targets on a background texture gradient: Is detection guided by discontinuities in the retinal-size gradient of items? Perception 30, 21-48.

Gregory, R. L. (1966). Eye and Brain. McGraw-Hill, New York.

Helmholtz, H. L. F., von (1896). Handbuch der Physiologischen Optik. Voss, Hamburg.

Humphreys, G. W., Keulers, N. and Donnelly, N. (1994). Parallel visual coding in three dimensions, Perception 23, 453-470.

Kleffner, D. A. and Ramachandran, V. S. (1992). On the perception of shape from shading, Perception and Psychophysics 52, 18-36.

Koch, C. and Ullman, S. (1985). Shifts in selective visual attention: Towards the underlying neural circuitry, Human Neurobiology 4, 219-227.

Müller, H. J. and Busch, A. (2003). Visual search for size-defined targets is modulated by the Ebbinghaus illusion: Facilitatory and inhibitory effects of the context objects (unpublished manuscript).

Müller, H. J., Heller, D. and Ziegler, J. (1995). Visual search for singleton feature targets within and across feature dimension, Perception and Psychophysics 57, 1-17.

Müller-Lyer, F. C. (1889). Optische Urteilstäuschungen, Archive für die Physiologie, Supplement, 263-270.

Nakagawa, D. (1958). Müller-Lyer illusion and retinal induction, Psychologia 1, 167-174.

Nakayama, K. and Silverman, G. H. (1986). Serial and parallel processing of visual feature conjunctions, Nature 320, 264-265.

Neisser, U. (1967). Cognitive Psychology. Appleton-Century-Crofts, New York.

Nothdurft, H. C. (1992). Feature analysis and the role of similarity in preattentive vision, Perception and Psychophysics 52, 355-375.

Nothdurft, H. C. (1993). The role of features in preattentive vision: comparison of orientation, motion and color cues, Vision Research 33, 1937-1958.

Quinlan, P. T. and Humphreys, G. W. (1987). Visual search for targets defined by combinations of color, shape, and size: An examination of the task constraints on feature and conjunction searches, Perception and Psychophysics 41, 455-472.

Ramachandran, V. S. (1988). Perception of shape from shading, Nature 331, 163-165.

Ratcliff, R., Van Zandt, T. and McKoon, G. (1999). Connectionist and diffusion models of reaction time, Psychol. Rev. 106, 261-300.

Rensink, R. A. and Enns, J. T. (1995). Preemption effects in visual search: Evidence for low-level grouping, Psychol. Rev. 102, 101-130.

Robinson, J. O. (1998). The Psychology of Visual Illusion. Dover Publications, Mineola, New York.

Stuart, G. W., Bossomaier, T. R. J. and Johnson, S. (1993). Preattentive processing of object size: Implications for theories of size perception, Perception 22, 1175-1193.

Treisman, A. (1988). Features and objects: The Fourteenth Bartlett Memorial Lecture, Quart. J. Exper. Psychol. 40A, 201-237.

Treisman, A. M. and Gelade, G. (1980). A feature-integration theory of attention, Cognitive Psychology 12, 97-136.

Treisman, A. and Gormican, S. (1988). Feature search in early vision: Evidence from search asymmetries, Psychol. Rev. 95, 14-48.

Wolfe, J. M. (1998). Visual search, in: Attention, Pashler, H. (Ed.), pp. 13-73. Psychology Press, Hove, UK.

Wolfe, J. M. (2003). Moving towards solutions to some enduring controversies in visual search, $\underline{\text { Trends }}$ in Cognitive Science 7, 70-76. 